\title{
Stigmatisierungserfahrungen von Menschen mit Diabetes mellitus in der Schweiz
}

\author{
Daniel Gredig • Annabelle Bartelsen-Raemy
}

Online publiziert: 17. November 2017

(C) Der/die Autor(en) 2017. Dieser Artikel ist eine Open-Access-Publikation.

Zusammenfassung Obwohl lange angenommen wurde, Menschen mit Diabetes mellitus seien nicht von Stigmatisierung betroffen, mehren sich in der Literatur die Hinweise auf Diskriminierung und stereotype Zuschreibungen. Allerdings liegen noch keine Studien vor, die diese Erfahrungen umfassend und konkret zugänglich machen. Um den Professionellen der Sozialen Arbeit, aber auch Angehörigen anderer Gesundheitsberufe weiterführende Erkenntnisse hierzu anbieten zu können, ging diese Studie der Frage nach, ob und in welchen Kontexten Menschen mit Diabetes, die in der Schweiz leben, Diskriminierung erfahren.

Schlüsselwörter Diabetes $\cdot$ Stigma $\cdot$ Stigmatisierung $\cdot$ Diskriminierung · Stereotype

\section{Experienced and perceived stigma of people living with Diabetes Mellitus in Switzerland}

\begin{abstract}
Despite the prevailing perception that diabetes mellitus is not a stigmatized condition, there is growing evidence of stigma. However, there have been no in-depth studies of stigma towards people living with diabetes (PWD). In order to complement the respective knowledge base of social workers and other health-allied professions, the present study aimed to establish whether PWD in Switzerland experience stigma, and if so, in what context and to what extent. The findings suggest that social work interventions should consider stigma explicitly and include designing strategies to reduce discrimination against PWD, and correct stereotypes about it.
\end{abstract}

Prof. Dr. D. Gredig $(\bowtie) \cdot$ A. Bartelsen-Raemy

Hochschule für Soziale Arbeit, Fachhochschule Nordwestschweiz,

Riggenbachstraße 16, CH-4600 Olten, Schweiz

E-Mail: daniel.gredig@fhnw.ch 
Keywords Diabetes $\cdot$ Stigma $\cdot$ Discrimination $\cdot$ Stereotypes

\section{Einleitung}

Menschen mit Diabetes mellitus sehen sich mit einer Reihe von spezifischen Belastungen konfrontiert. Diese ergeben sich nicht nur aus der chronischen Krankheit selbst oder aus den Folgekrankheiten, die sich je nach Behandlungserfolg in unterschiedlichem Ausmaß zusätzlich einstellen können. Auch das Krankheitsmanagement, das Menschen mit dieser Diagnose auf sich nehmen müssen, oder die medizinisch geforderten Veränderungen ihrer Lebensweise hinsichtlich Ernährung und Bewegung können den Betroffenen zur Belastung werden und eine selbstbestimmte Lebensführung zusätzlich beeinträchtigen.

Soziale Arbeit, die in die Beratung und Unterstützung von Menschen mit Diabetes in verschiedenen Lebenswelten und in unterschiedlichen Lebensphasen eingebunden ist, fokussiert in der Regel auf diese psychosozialen Aspekte. Ihre Angebote zielen auf die Erhaltung bzw. Wiedergewinnung einer selbstbestimmten Lebensführung mit - und in gewisser Weise trotz - Diabetes und die Förderung von Wohlbefinden. Im Rahmen interprofessioneller Teams tragen Sozialarbeitende dazu bei, die Betroffenen beim Krankheitsmanagement und der Realisierung der medizinisch geforderten Veränderungen ihrer Lebensweise zu bestärken, die dafür notwendigen Ressourcen zu erschliessen und soziale Unterstützung zu fördern. Darüber hinaus unterstützen sie die Betroffenen dabei, ihre Krankheit zu akzeptieren, und die Belastungen zu bewältigen, die sich aus der Diagnose, dem Krankheitsmanagement und den geforderten Veränderungen der Lebensweise ergeben können (Voseckova et al. 2017; Ciporen 2012; DeCoster und Cummings 2005; DeCoster 2001).

Der Stress, dem sich Menschen mit Diabetes ausgesetzt sehen, und die Frustrationen, die vom Krankheitsmanagement ausgehen, das ihnen abgefordert wird, wie auch die emotionalen Belastungen, werden gängiger Weise mit dem Begriff „Diabetes Distress“" gefasst und sind seit langem Gegenstand von Forschung (vgl. z. B. Wardian und Sun 2014; Balfe et al. 2013; Egede und Dismuke 2012). Im Kontrast hierzu hat ein anderer psychosozialer Aspekt des Lebens mit Diabetes bislang kaum Beachtung gefunden: Dass Menschen mit Diabetes auch nachteiligen sozialen Reaktionen ausgesetzt und von negativen Zuschreibungen und ungerechtfertigter Ungleichbehandlung, von Benachteiligung und Ausschluss betroffen sein können, ist bis vor kurzem selten aufgegriffen worden und darf alles andere als durchgängig anerkannt gelten (Benedetti 2014). In der Literatur zur Sozialen Arbeit mit Menschen mit Diabetes ist dieser Aspekt bis auf wenige Anklänge (vgl. z. B. bei Ciporen 2012, S. 29) unbeachtet geblieben.

Aus sozialwissenschaftlicher Perspektive und unter Bezugnahme auf die „Epistemologie“ (Winkler 2003) der Sozialen Arbeit kann diese Dynamik, der sich Menschen mit Diabetes ausgesetzt sehen, als Stigmatisierung (Goffman 1963) verstanden werden, die in manifesten stereotypen Zuschreibungen und in ungerechtfertigter Ungleichbehandlung - und damit in Diskriminierung - ihren Ausdruck findet (Pescosolido et al. 2008; Link und Phelan 2001). Der Diskriminierung und den Stereotypen, die sich an Diabetes mellitus festmachen, ist erst in den letzten Jah- 
ren eine etwas lebhaftere Aufmerksamkeit zuteilgeworden. Gesichertes Wissen ist entsprechend gering (Schabert et al. 2013). So hält etwa eine neuere, große internationale Studie zu psychosozialen Aspekten von Diabetes lediglich pauschal fest, dass Menschen mit Diabetes davon berichten, schon einmal diskriminiert worden zu sein (Wens et al. 2013). Im letzten Jahrzehnt vereinzelt erschienene qualitative Studien zeigten konkret auf, dass Menschen mit Diabetes die Erfahrung gemacht hatten, gemieden $\mathrm{zu}$ werden, und dass sie sich beschwerten, dass sie für die Erneuerung ihrer Fahrerlaubnis ein ärztliches Gutachten beibringen mussten (Broom und Whittaker 2004). Betroffene verweisen auch auf Einschränkungen bei Reisen, zerbrochene Freundschaften und z. B. auch auf die Erfahrung, wegen Diabetes von der Adoption eines Kindes ausgeschlossen worden zu sein (Browne et al. 2013). Zudem berichten Menschen mit Diabetes von der Erfahrung, auf dem Arbeitsmarkt ungerechtfertigt ungleich behandelt zu werden. Die Untersuchungen hierzu - dem einzigen Aspekt, der schon länger Gegenstand von Forschung ist - lassen deutlich werden, dass ein Teil der Menschen mit Diabetes in ihrem bisherigen Leben bereits einmal erlebt hat, dass sie wegen Diabetes nicht angestellt wurden, ihre Anstellung verloren, nicht befördert wurden, keine Lohnerhöhung erhielten oder Probleme mit Versicherungen hatten (Tak-Ying Shiu et al. 2003; Petrides et al. 1995; Griffith und Moses 1993; Robinson et al. 1990; Songer et al. 1989). Piera Nebiker-Pedrotti und Kolleg*innen (2009) bestätigen diese Befunde auch mit Blick auf die Schweiz. Sie berichten davon, dass Menschen mit Diabetes aufgrund ihrer Krankheit gekündigt wurden, eine Stelle nicht erhielten oder Probleme mit Versicherungen hatten.

Menschen mit Diabetes vermerken auch stereotype Zuschreibungen. So nehmen Typ 2 Diabetiker*innen wahr, als ,schwach“, ,fett“, ,träge“, „faul“, ,verfressen“ oder „Vielfrasse“ zu gelten (Tak-Ying Shiu et al. 2003). Andere Stereotype präsentieren sie als ,armselig“, „wenig intelligent“ oder als „,schlechte Menschen“ (Browne et al. 2013). Menschen mit Diabetes erfahren, dass sie für ihre Krankheit selbst verantwortlich gemacht werden. Solche Schuldzuschreibungen werden von Menschen mit Diabetes Typ 2 wahrgenommen (Browne et al. 2013), sind aber auch als Aussagen über junge Menschen mit Diabetes Typ 1 dokumentiert (Vishwanath 2014). Insulininjizierende Personen machen zudem die Erfahrung, mit injizierenden Drogenkonsumierenden verwechselt zu werden (Tak-Ying Shiu et al. 2003).

Der Blick auf den Forschungsstand lässt mithin erkennen, dass sich negative soziale Reaktionen an der Krankheit an sich festmachen, aber auch an ihren Folgeerscheinungen - seien sie nun punktueller Natur (wie Hypoglykämien unterschiedlicher Schwere) oder progressiver Natur (wie Folgekrankheiten) - und an Elementen des Krankheitsmanagements (wie z. B. dem Injizieren von Insulin). Es zeigt sich aber auch, dass weniger markante Formen von ungerechtfertigter Ungleichbehandlung als jene, die im Erwerbsleben erfolgen, bislang nicht systematisch untersucht worden sind. Zudem beziehen sich die vorliegenden Hinweise zumeist auf Erfahrungen von Menschen mit Diabetes, die im angelsächsischen Raum oder China leben.

Da davon auszugehen ist, dass die Erfahrungen von Stigmatisierung aber nicht einfach kontextunabhängig sind, sondern vielmehr von kulturellen (Schabert et al. 2013), sozialen, politischen und auch rechtlichen Bedingungen mit geprägt sind, ist einer Verallgemeinerung der dokumentierten Stigmatisierungserfahrungen über den 
ursprünglichen Kontext hinaus mit Vorsicht zu begegnen. Vielmehr bedarf es einer Untersuchung der Erfahrungen von Menschen mit Diabetes in unterschiedlichen nationalstaatlichen Kontexten. Und wie Jessica L. Browne und Kolleg*innen (2013) in weiterer Konkretisierung dieses Forschungsdesiderats hervorheben, sind zunächst vertiefte Explorationen der Wahrnehmungen und Erfahrungen von diabetes-bezogenen Stigmatisierungen aus der Perspektive der Betroffenen gefragt, da diese den Ausgangspunkt für ein Forschungsprogramm in diesem Bereich darstellen.

\section{Ziel und Fragestellung der Untersuchung}

Die vorliegende Studie möchte dazu beitragen, die Wissenslücke hinsichtlich der Stigmatisierungserfahrungen von Menschen mit Diabetes zu schliessen und die Wissensbasis der Sozialen Arbeit in diesem Feld zu erweitern, indem sie sich der Erfahrungen von Menschen mit Diabetes in der Schweiz zuwendet und dabei der Perspektive der Menschen mit Diabetes folgt und deren Erfahrung und Erleben erfasst. Dies im Wissen darum, dass die Erkenntnisse, die sich aus der Erforschung subjektiv erlebter Diskriminierung ergeben, nicht umstandslos in Aussagen über (objektiv) messbare Teilhabechancen überführen lassen (El-Mafaalani et al. 2017). Vor diesem Hintergrund exploriert diese Untersuchung, welche Stigmatisierungserfahrungen die in der Schweiz wohnhaften Diabetiker*innen machen.

In einem ersten Zugriff fokussiert sie auf die Lebenszeitprävalenz von Stigmatisierungserfahrungen. Mit Blick auf Diskriminierungserfahrungen ging sie konkret den Fragen nach, ob und in welchen Kontexten und Situationen Menschen mit Diabetes Erfahrungen von Diskriminierung machen und worin diese zum Ausdruck gelangen; wie viele Menschen mit Diabetes diese Erfahrungen schon machten und in wie vielen Kontexten sie mit Diskriminierung konfrontiert waren, und inwiefern es für Diskriminierung besonders vulnerable Gruppen gibt. Mit Blick auf die wahrgenommenen stereotypen Zuschreibungen fragte sie danach, welche stereotypen Zuschreibungen Menschen mit Diabetes wahrnehmen und ob sich in den wahrgenommenen Stereotypen bestimmte Muster erkennen lassen. In einem zweiten Zugriff fokussierte die Studie auf die Gegenwartsnähe von Diskriminierungserfahrungen und ging der Frage nach, ob und welche Diskriminierungserfahrungen Menschen mit Diabetes in den letzten zehn Jahren vor der Befragung, konkret im Zeitraum von 2003 bis 2013 gemacht hatten. In einem dritten Zugriff rückte die Studie die Unmittelbarkeit der Diskriminierungserfahrungen ins Zentrum und untersuchte, ob und welche Diskriminierungserfahrungen Menschen mit Diabetes bereits im ersten Jahr nach der Diagnose machten.

Diese Fragestellung thematisiert die Erfahrungen in unterschiedlichen Situationen und Kontexten mit Blick auf das gesamte Leben mit Diabetes (Lebenszeitprävalenz), die letzten zehn Jahre und schließlich das erste Jahr nach der Diagnose. Damit lässt sie die Frequenz außer Acht, mit der sich eine Erfahrung in einer bestimmten Situation wiederholt. Auf eine Erhebung der Häufigkeit der Wiederholungen von spezifischen Erfahrungen in einer bestimmten Situation wurde in dieser Exploration bewusst verzichtet. Die Situationen sind so unterschiedlich, dass die einen täglich vorkommen können, während sich andere nur einmal im Leben ergeben mögen. 
Einmalige Erfahrungen können aber für die Lebensführung genauso entscheidend und hinsichtlich Chancengleichheit schwerwiegender und für das weitere Leben prägender sein (z. B. im Rahmen der Berufswahl) als Erlebnisse, die sich täglich wiederholen, unangebracht und beeinträchtigend sind, aber keine weitreichenden Konsequenzen haben (z. B. eine bevormundende Einmischung von Kolleg*innen beim Essen). Auf dem aktuellen Stand der Forschung schien es wichtiger, die konkreten Situationen und Kontexte von Ungleichbehandlung in Erfahrung zu bringen und die Frequenz einer bestimmten Art von Diskriminierung zurückzustellen.

\section{Methode}

\subsection{Design}

Da zu den meisten Bereichen kein Vorwissen bestand, wurde ein sequenzielles exploratives Design gewählt (Creswell 2009, S. 206f.), in dem Verfahren der qualitativen und quantitativen Sozialforschung in dieser Abfolge miteinander verbunden wurden.

In der qualitativen, vorausgehenden Untersuchung wurden die Kontexte von Diskriminierung ermittelt und die Stereotype erfasst, die Menschen mit Diabetes wahrgenommen hatten. Hierzu wurden problemzentrierte Interviews (Witzel 1985) mit einem maximal diversen Sample (Patton 2002) von 30 Frauen und Männern mit Diabetes Typus 1 und 2 geführt. Die Interviewdaten wurden mittels theoretischem Kodieren (Strauss und Corbin 1996) ausgewertet. So konnten unter anderem 33 Situationen erfasst werden, in denen Menschen mit Diabetes wegen ihrer Krankheit schon einmal eine ungerechtfertigte Ungleichbehandlung erfahren hatten. Diese Situationen konnten sieben Kontexten zugeordnet werden: Schule, Erwerbstätigkeit, Armee/Militärdienst, Mobilität, Steuern, Versicherungen, Freizeit und soziale Kontakte. Weiter resultierte eine Zusammenstellung mit 26 stereotypen Zuschreibungen, welche die Befragten wahrgenommen hatten (Raemy et al. 2012). Die anschließende quantitative Studie, die in diesem Beitrag im Zentrum steht, wurde als Querschnittsuntersuchung bei Menschen mit Diabetes in der Schweiz konzipiert. Sie baut auf den Ergebnissen der qualitativen Studie auf und legte diese der Entwicklung der Instrumente für die standardisierte Datengenerierung zugrunde. Die Datenerhebung erfolgte mittels eines anonymen, schriftlichen, selbst auszufüllenden Fragebogens.

\subsection{Operationalisierung, Messinstrumente und Konstruktion des Erhebungsinstruments}

Zur Erhebung von Stigmatisierungserfahrungen wurden zwei formative Indizes entwickelt, die aus einer Reihe von Einzelitems bestehen (vgl. Diamantopoulos und Siguaw 2006; Schnell et al. 1999). Der erste Index dient zur Erfassung der erfahrenen Diskriminierung. Damit werden Situationen erfasst, in denen Personen mit Diabetes aus ihrer Warte betrachtet diabetesbedingt eine ungerechtfertigte Ungleichbehandlung erfahren haben. Zu jeder Situation, die aus der qualitativen Vorstudie bekannt war, wurde ein Item ausgearbeitet. Zunächst wurde - gestützt auf die theoretische Kodierung (Strauss und Corbin 1996), die im Zuge der qualitativen Auswertung der 
verbalen Daten vorgenommen worden war - die Textpassage identifiziert, in der die Erfahrung geschildert wird. Aus dieser Beschreibung wurde die Formulierung herausgegriffen, die das Ereignis am kürzesten fasste. Diese Aussage wurde von indexikalen Anteilen (zum Beispiel Angaben zu Zeit, Ort, Personen) befreit und in einem nächsten Schritt abstrahierend reformuliert. Falls sachlich korrekt, wurde bei der Formulierung des Items auf die ursprüngliche umgangssprachliche Wortwahl der Befragten aus den qualitativen Interviews zurückgegriffen. Die einzelnen Items wurden nach Kontexten geordnet und mit Antwortkategorien versehen. Dieser Index der Diskriminierungserfahrung umfasst 33 Items. Er ist so formuliert, dass die Frage die Form eines ersten Teilsatzes hat, der dann sinnhaft von jedem der Items zu einem vollständigen Fragesatz ergänzt wird. Die Frage lautete: „Haben Sie schon einmal die Erfahrung gemacht ....“. Ein weiterführendes Item lautete zum Beispiel: ,,... dass Ihnen von Vorgesetzten wegen Diabetes eine leitende Funktion entzogen wurde?“ Die Antwortkategorien lauten ,,ja“, ,,nein“, „,trifft nicht zu, weil ich nie eine leitende Funktion hatte" und ,trifft nicht zu, weil ich damals noch keinen Diabetes hatte“.

Der zweite Index dient zur Erfassung der wahrgenommenen Stereotype gegenüber Menschen mit Diabetes. Er wurde nach demselben Verfahren entwickelt und umfasst 26 Items. Auch hier wird die Frage durch einen Teilsatz eingeleitet, der durch die Items zu einer vollständigen Frage komplettiert wird. Die Frage lautet: „Hatten Sie schon einmal das Gefühl, dass ...“. Ein weiterführendes Item lautet zum Beispiel: ,,... Leute denken, Menschen mit Diabetes seien bemitleidenswert?“ Die Antwortkategorien lauten ,ja“" und ,nein“.

Um prüfen zu können, ob die Erfahrungen mit diesem Instrument valide erfasst werden können, wurden die zwei Fragebatterien den Interviewpartner*innen der vorangegangenen qualitativen Studie zur Beantwortung zugestellt. Die Antworten auf die Items in den zwei Indizes wurden mit den Aussagen im Interview verglichen. Der Vergleich zeigte, dass die konstruierten Instrumente sowohl die Diskriminierungserfahrungen als auch die wahrgenommenen Zuschreibungen in Übereinstimmung mit den Angaben der Befragten im Interview abzubilden vermochten.

Im Weiteren erhob der Fragebogen Angaben zum Diabetes-Typ, das Alter bei der Diagnose und soziodemografische Merkmale. Dabei gelangten Instrumente zum Einsatz, die für eine Erhebung dieser Merkmale in der Schweiz angemessen sind.

Der Fragebogen wurde so aufgebaut, dass die Indizes zur Diskriminierungserfahrung und Wahrnehmung von Stereotypen an dritter und vierter Stelle standen und damit im Bereich lagen, bei dem von einer hohen Konzentration der Befragten ausgegangen werden darf (Scherpenzeel und Saris 1997; Költringer 1993). Der Fragebogen wurde in zwei gleichförmigen Fassungen in Deutsch und Französisch erstellt. Hierzu wurde die deutsche Version in einem Verfahren von Übersetzung und Rückübersetzung in eine französische Fassung gebracht. Das Layout der beiden Sprachfassungen war identisch. Es wurde mit der Software zum Einlesen der Fragebogen abgestimmt, und jede Seite wurde mit Markierungen versehen, die eine sichere Erfassung der Daten mit Scanner erlauben. Schließlich wurden die Fragebogen in einem Pretest mit einem Sample von 220 (108 deutschsprachigen und 112 französischsprachigen) Mitgliedern der Schweizerischen Diabetesgesellschaft auf Verständlichkeit, Handhabung und Sachangemessenheit geprüft. 


\subsection{Stichprobengewinnung}

Mangels rechtlicher Grundlagen führt die Schweiz kein Krankheitsregister, das eine Beschreibung der Grundgesamtheit von Menschen mit Diabetes in der Schweiz zuliesse. Versuche, die Anzahl von Menschen mit Diabetes zu bestimmen und diese Population zu beschreiben, stützen sich auf unterschiedliche Quellen und führen nicht weiter als zu Schätzungen (vgl. z. B. Huber et al. 2014). Dieser Umstand lässt eine Zufallsstichprobe wenig effektiv und ein Quotensample unmöglich werden. Vor diesem Hintergrund wurde ein convenience sample von Menschen mit Diabetes aus der deutsch- oder französischsprachigen Schweiz gebildet. Um eine möglichst große Anzahl von Betroffenen in unterschiedlichen Lebenslagen möglichst direkt - also unabhängig von der Mitwirkungsbereitschaft von Intermediären, wie zum Beispiel Ärzt*innen oder Berater*innen - erreichen zu können, wurden alle Privatpersonen angesprochen, welche das Magazin „d-journal“ bzw. das „d-journal romand" abonniert haben. Die frei zugänglichen Magazine zählten 2013 insgesamt 25.398 Abonnent*innen, davon 21.154 Privatpersonen, zu denen alle Mitglieder der Schweizerischen Diabetes-Gesellschaft wie auch weitere Interessierte zählten.

Der Fragebogen und das frankierte und adressierte Antwortcouvert wurden mit der Ausgabe des „d-journal“ bzw. des „d-journal romand“ vom Oktober 2013 verschickt. Eine Mahnung oder eine erneute Zustellung der Unterlagen waren aufgrund des gewählten Wegs der Ansprache nicht möglich. Es gingen insgesamt 3401 Fragebogen ein, was einem unter diesen Umständen erwartenden Rücklauf (Dillman 1978) von rund $16 \%$ entspricht.

\subsection{Datenerfassung und Auswertung}

Die Fragebogen wurden einzeln per Scanner eingelesen und als Bilddatei abgelegt. Die Bilddateien wurden (mit Ausnahme des Kommentarfeldes, das zum Teil personenidentifizierende Angaben enthielt) von der Firma Electronic Paper mit der Software TELEForm per OCR erfasst und in Excel-Dateien abgelegt. Die Daten wurden in das Statistik-Softwarepaket IBM SPSS 22 eingelesen. Die Auswertung der Daten erfolgte mittels Verfahren der beschreibenden Statistik. Zur Ermittlung von Zusammenhängen wurden dem Skalenniveau entsprechende bivariate und multivariate Verfahren, konkret multiple Regressionsanalysen eingesetzt. Die Ermittlung der Muster unter den wahrgenommenen Zuschreibungen erfolgte mittels einer Faktorenanalyse (mit Maximum-Likelihood-Methode und schiefwinkliger Rotation) (Osborne und Costello 2009). Zur Ermittlung der Erfahrungen in den letzten zehn Jahren vor der Befragung wurde so vorgegangen, dass nur die Angaben jener Befragten in die betreffende Analyse einbezogen wurden, deren Diabetesdiagnose zum Zeitpunkt der Befragung nicht mehr als zehn Jahre zurücklag und damit ausschließlich von Erfahrungen aus dem Zeitraum zwischen 2003 und 2013 berichten konnten. Auf die gleiche Weise wurde verfahren, um die Erfahrungen zu ermitteln, die ein Jahr vor der Befragung gemacht wurden. Es wurden hierzu nur jene Befragten in die Analyse beinbezogen, die ein Jahr oder weniger mit Diabetes lebten. 


\section{Ergebnisse}

Es konnten Daten von 3347 Personen mit Diabetes in die Auswertung einbezogen werden. Die Zusammensetzung der Stichprobe hinsichtlich der wichtigsten diabetesbezogenen und soziodemografischen Merkmale der beteiligten Menschen mit Diabetes geht aus Tab. 1 hervor.

Das Alter, in dem den Befragten Diabetes diagnostiziert wurde, zeigt eine weite Spanne. Bei einigen der Befragten wurde Diabetes bereits im ersten Lebensjahr diagnostiziert. Bei anderen wurde die Diagnose erst in einem Alter über 80 Jahren gestellt. Der Median des Alters bei der Diagnose liegt bei 45 Jahren. Die relativ große Anzahl von 233 fehlenden Angaben zum Zeitpunkt der Diagnose zeigt allerdings auf, dass sich ein Teil der Befragten nicht mehr genau zu erinnern vermag, in welchem Alter ihnen die Diabetesdiagnose gestellt wurde. Diesem Befund

Tab. 1 Diabetesbezogene und soziodemografische Merkmale der Befragten $(N=3347)$

\begin{tabular}{|c|c|c|c|c|}
\hline Merkmal & & $n$ & $\%$ & $N$ \\
\hline \multirow[t]{2}{*}{ Diabetes-Typ } & Typ 1 & 1352 & 42,3 & 3193 \\
\hline & Typ 2 & 1841 & 57,7 & \\
\hline \multirow[t]{8}{*}{ Alter } & 16-25 Jahre & 108 & 3,4 & 3159 \\
\hline & 26-35 Jahre & 144 & 4,6 & \\
\hline & 36-45 Jahre & 218 & 6,9 & \\
\hline & 46-55 Jahre & 448 & 14,2 & \\
\hline & 56-65 Jahre & 646 & 20,4 & \\
\hline & 66-75 Jahre & 934 & 29,6 & \\
\hline & 76-85 Jahre & 573 & 18,1 & \\
\hline & $>85$ Jahre & 88 & 2,8 & \\
\hline \multirow[t]{2}{*}{ Geschlecht } & Frauen & 1479 & 45,2 & 3270 \\
\hline & Männer & 1791 & 54,8 & \\
\hline \multirow{9}{*}{$\begin{array}{l}\text { Formales } \\
\text { Bildungsniveau }\end{array}$} & Kein Schulabschluss & 10 & 0,4 & 3265 \\
\hline & Primarschule & 145 & 4,4 & \\
\hline & Obligatorische Schule & 305 & 9,3 & \\
\hline & $\begin{array}{l}\text { Mittelschule, Gymnasium, Berufsma- } \\
\text { turität }\end{array}$ & 154 & 4,7 & \\
\hline & Lehrkräfteseminar & 110 & 3,4 & \\
\hline & Berufslehre, Vollzeitberufsschule & 1299 & 39,8 & \\
\hline & Höhere Fach- und Berufsausbildung & 571 & 17,5 & \\
\hline & Höhere Fachschule & 243 & 7,4 & \\
\hline & Universität/Fachhochschule & 428 & 13,1 & \\
\hline \multirow{7}{*}{$\begin{array}{l}\text { Erwerbs- bzw. } \\
\text { Beschäftigungssituation }^{\mathrm{a}}\end{array}$} & Erwerbstätigkeit Vollzeit & 636 & 16,3 & 3913 \\
\hline & Erwerbstätigkeit Teilzeit & 626 & 16,0 & \\
\hline & Studium & 57 & 1,5 & \\
\hline & Erwerbslosigkeit & 50 & 1,3 & \\
\hline & Pension & 1701 & 43,5 & \\
\hline & Arbeitsunfähigkeit & 158 & 4,0 & \\
\hline & Hausfrau/Hausmann & 685 & 17,5 & \\
\hline
\end{tabular}


Tab. 1 Diabetesbezogene und soziodemografische Merkmale der Befragten $(N=3347)$ (Fortsetzung)

\begin{tabular}{|c|c|c|c|c|}
\hline Merkmal & & $n$ & $\%$ & $N$ \\
\hline \multirow[t]{3}{*}{ Leitungsfunktion } & Direktionsfunktion & 169 & 12,2 & 1381 \\
\hline & Kaderfunktion & 339 & 24,5 & \\
\hline & Keine Direktions- oder Kaderfunktion & 873 & 63,2 & \\
\hline \multirow[t]{4}{*}{ Zivilstand } & Ledig & 518 & 16,0 & 3239 \\
\hline & Verheiratet & 1924 & 59,4 & \\
\hline & Eingetragene Partnerschaft & 54 & 1,7 & \\
\hline & Verwitwet, geschieden, getrennt & 743 & 22,9 & \\
\hline \multirow[t]{5}{*}{ Wohnform ${ }^{\mathrm{a}}$} & Alleine & 812 & 22,3 & 3636 \\
\hline & Mit Partner/in & 2188 & 60,2 & \\
\hline & Mit Kindern & 467 & 12,8 & \\
\hline & Mit Verwandten & 124 & 3,4 & \\
\hline & In einer Institution & 45 & 1,3 & \\
\hline \multirow[t]{2}{*}{ Sprachregion } & Deutschschweiz & 2487 & 74,3 & 3347 \\
\hline & Romandie & 860 & 25,7 & \\
\hline \multirow[t]{2}{*}{ Nationalität } & Schweiz & 3128 & 95,3 & 3282 \\
\hline & Andere & 154 & 4,7 & \\
\hline
\end{tabular}

${ }^{a}$ Mehrfachantworten; Prozentangaben beziehen sich auf die Anzahl Befragter

entsprechend, schauten die Befragten zum Zeitpunkt der Befragung auf recht unterschiedlich lange Lebenszeiten mit Diabetes zurück. Die Spanne reicht von einer Diagnose im Jahr der Befragung bis zu einer Diagnose, die 76 Jahre zurückliegt. Im Durchschnitt leben die Befragten gut 21 Jahre mit Diabetes. Der Median der Lebenszeit mit Diabetes liegt bei 19 Jahren.

\subsection{Erfahrung von ungerechtfertigter Ungleichbehandlung}

Ein knappes Drittel der Befragten (31,5\%) gab an, dass sie bislang keine der aufgeführten Situationen ungerechtfertigter Ungleichbehandlung erfahren haben. 68,5\% der Befragten gibt hingegen an, dass sie wegen Diabetes schon diskriminiert worden sind. Tab. 2 zeigt auf, wie viele Befragte mit Diabetes in eine bestimmte Situation gelangt sind und die verzeichnete Form von ungerechtfertigter Ungleichbehandlung aufgrund von Diabetes erlebt haben.

Tab. 2 Diskriminierungserfahrungen: Situationen und Kontexte (Lebenszeitprävalenz) $(N=3347)$

\begin{tabular}{llll}
\hline Erfahrene ungerechtfertigte Ungleichbehandlung $^{\mathrm{a}}$ & $N^{\mathrm{b}}$ & $n^{\mathrm{c}}$ & $\%^{\mathrm{d}}$ \\
\hline Kontext Freizeitgestaltung und sozialer Kontakt & & & \\
Ungerechtfertigte Andersbehandlung im Bekanntenkreis & 3139 & 379 & 12,1 \\
Sich beim Essen mit Freunden ständig rechtfertigen müssen & 3144 & 767 & 24,4 \\
Verhinderung der Ausübung einer gewünschten Sportart & 2453 & 385 & 15,7 \\
Verweigerung des Zugangs zu Ausgehlokalen & 2886 & 32 & 1,1 \\
Kontext Schule & & & \\
Ausschluss von Schulausflügen & 1430 & 103 & 7,2 \\
\hline
\end{tabular}


Tab. 2 Diskriminierungserfahrungen: Situationen und Kontexte (Lebenszeitprävalenz) $(N=3347)$ (Fortsetzung)

\begin{tabular}{|c|c|c|c|}
\hline Erfahrene ungerechtfertigte Ungleichbehandlung ${ }^{\mathrm{a}}$ & $N^{\mathrm{b}}$ & $n^{\mathrm{c}}$ & $\%^{\mathrm{d}}$ \\
\hline \multicolumn{4}{|l|}{ Kontext Erwerbstätigkeit } \\
\hline Unfreiwillige Aufgabe eines Berufsziels & 1956 & 221 & 11,3 \\
\hline Verweigerung der anvisierten Berufsausbildung & 1851 & 161 & 8,7 \\
\hline Ablehnung bei Stellenbewerbung & 2090 & 167 & 8,0 \\
\hline Entzug einer leitenden Funktion & 1773 & 67 & 3,8 \\
\hline Entzug einer leitenden Funktion wegen einer Hypoglykämie & 1798 & 41 & 2,3 \\
\hline Empfehlung besonderer Arbeitszeiten & 2476 & 91 & 3,7 \\
\hline Entzug interessanter beruflicher Tätigkeiten & 2492 & 106 & 4,3 \\
\hline Verweigerung einer Beförderung & 2463 & 87 & 3,5 \\
\hline Angst und Zurückhaltung seitens der Arbeitskolleg*innen & 2584 & 369 & 14,3 \\
\hline Einmischung in Essgewohnheiten seitens der Arbeitskolleg*innen & 2602 & 482 & 18,5 \\
\hline Aufforderung zur Kündigung & 2460 & 81 & 3,3 \\
\hline Entlassung & 2481 & 65 & 2,6 \\
\hline Entlassung und Antrag auf IV-Rente nahegelegt & 2481 & 74 & 3,0 \\
\hline Aufforderung zur Frühpensionierung & 2478 & 118 & 4,8 \\
\hline \multicolumn{4}{|l|}{ Kontext Militär } \\
\hline Bei der Aushebung für dienstuntauglich erklärt & 753 & 252 & 33,5 \\
\hline Bei der Aushebung für zivilschutzuntauglich erklärt & 800 & 106 & 13,3 \\
\hline Aus der Armee entlassen & 819 & 197 & 24,1 \\
\hline $\begin{array}{l}\text { Verpflichtung zu Wehrpflichtersatz trotz Bereitschaft, Militärdienst zu } \\
\text { leisten }\end{array}$ & 885 & 272 & 30,7 \\
\hline $\begin{array}{l}\text { Verpflichtung zu Wehrpflichtersatz trotz Bereitschaft, Zivilschutz zu } \\
\text { leisten }\end{array}$ & 911 & 210 & 23,1 \\
\hline \multicolumn{4}{|l|}{ Kontext Mobilität } \\
\hline Sonderbehandlung und -prüfung beim Sicherheitscheck am Flughafen & 2488 & 578 & 23,2 \\
\hline Auflage, ärztliche Atteste beizubringen, um die Fahrerlaubnis zu erhalten & 2456 & 327 & 13,3 \\
\hline \multicolumn{4}{|l|}{ Kontext Steuern } \\
\hline Ablehnung von Abzügen von Krankheitskosten seitens des Steueramtes & 2865 & 1090 & 38,0 \\
\hline \multicolumn{4}{|l|}{ Kontext Versicherungen } \\
\hline Verweigerung Lebensversicherung & 1576 & 451 & 28,6 \\
\hline Verweigerung Erwerbsausfallversicherung & 1529 & 190 & 12,4 \\
\hline Verweigerung Taggeldversicherung & 1687 & 223 & 13,2 \\
\hline $\begin{array}{l}\text { Verweigerung Zusatzversicherung zur obligatorischen Krankenversiche- } \\
\text { rung }\end{array}$ & 1927 & 509 & 26,4 \\
\hline Kündigung Zusatzversicherung zur obligatorischen Krankenversicherung & 2472 & 70 & 2,8 \\
\hline Leistungsvorbehalt der Pensionskasse & 2216 & 258 & 11,6 \\
\hline
\end{tabular}

${ }^{\mathrm{a} M e h r f a c h a n t w o r t e n ; ~}{ }^{\mathrm{b}}$ Anzahl Antwortende; ${ }^{\mathrm{c}}$ Befragte, die angeben, diese Erfahrung gemacht zu haben;

${ }^{\mathrm{d}}$ Anteil an den Antwortenden

21,4 \% der Befragten führen Erfahrungen aus einer, 12,3\% aus zwei Situationen an. Die übrigen Befragten nennen Erfahrungen in mehreren Situationen. Betrachtet man jene Befragten, die überhaupt je eine Ungleichbehandlung wahrgenommen haben $(n=2245)$, liegt der Median der Erfahrungen von ungerechtfertigter Ungleichbehandlung bei drei Situationen. Zur Ermittlung besonders stark betroffener 
Gruppen von Menschen mit Diabetes wurde die Anzahl Situationen, in denen die Befragten schon eine Ungleichbehandlung wegen Diabetes erfahren haben, auf Zusammenhänge mit dem Diabetes-Typ sowie dem Geschlecht, dem Landesteil, der aktuellen Einbindung in die Erwerbstätigkeit, dem Alter und der Lebenszeit mit Diabetes hin betrachtet. Die multiple Regressionsanalyse zeigt dabei einen mittleren Zusammenhang (Cohen 1992) zwischen der Anzahl Situationen, in denen die Befragten Ungleichbehandlungen erfahren haben, und ihrem Alter $(\beta=-0,38$; $p \leq$ $0,001)$ und ihrer Lebenszeit mit Diabetes $(\beta=0,34 ; p \leq 0,001)$ auf. Je jünger sie sind und je länger sie schon mit Diabetes leben, desto mehr Situationen führen die Befragten an, in denen sie schon Ungleichbehandlung erlebt haben. Zudem zeigt sich ein schwacher Zusammenhang mit dem Geschlecht $(\beta=0,12 ; p \leq 0,001)$ sowie dem Diabetestyp $(\beta=-0,09 ; p \leq 0,001)$. Männer und Personen mit Diabetes Typ 1 haben von mehr Situationen berichtet, in denen sie schon einmal Ungleichbehandlung erfahren haben. Es zeigt sich jedoch kein systematischer Zusammenhang zwischen der Anzahl Situationen und dem Landesteil, in dem die Befragten leben, und ihrem Erwerbs- bzw. Beschäftigungsstatus ${ }^{1}$.

\subsection{Diskriminierungserfahrungen aus dem Zeitraum von 2003 bis 2013}

Betrachtet man die Angaben derjenigen Befragten, bei denen erst in den zehn Jahren vor der Befragung Diabetes diagnostiziert worden war $(n=706)$, erhält man einen Einblick in die Erfahrungen im Zeitraum von 2003 bis 2013. $41 \%$ der Befragten, die erst seit zehn Jahren Diabetes haben, gaben an, dass sie bislang keine der aufgeführten Situationen ungerechtfertigter Ungleichbehandlung erfahren haben. $59 \%$ der Befragten gibt hingegen an, dass sie wegen Diabetes schon ungerechtfertigt ungleich behandelt worden sind. Dabei zeigt sich, dass jene Befragten, die überhaupt nur auf Erfahrungen in diesem Zeitraum zurückblicken können, in sämtlichen verzeichneten Situationen Erfahrungen von ungerechtfertigter Ungleichbehandlung gemacht haben. Tab. 3 zeigt auf, wie viele dieser Befragten mit Diabetes in eine bestimmte Situation gelangt sind und die verzeichnete Form von ungerechtfertigter Ungleichbehandlung aufgrund von Diabetes erlebt haben.

Tab. 3 Diskriminierungserfahrungen in den Jahren von 2003 bis 2013: Situationen und Kontexte $(n=706)$

\begin{tabular}{lccc}
\hline Erfahrene ungerechtfertigte Ungleichbehandlung $^{\mathrm{a}}$ & $N^{\mathrm{b}}$ & $n^{\mathrm{c}}$ & $\%^{\mathrm{d}}$ \\
\hline Kontext Freizeitgestaltung und sozialer Kontakt & & & \\
Ungerechtfertigte Andersbehandlung im Bekanntenkreis & 653 & 83 & 12,7 \\
Sich beim Essen mit Freund*innen ständig rechtfertigen müssen & 661 & 195 & 29,5 \\
Verhinderung der Ausübung einer gewünschten Sportart & 535 & 76 & 14,2 \\
Verweigerung des Zugangs zu Ausgehlokalen & 592 & 6 & 1,0 \\
\hline
\end{tabular}

\footnotetext{
1 Weitere Angaben zum Regressionsmodell: $N=2867$; die standardisierten Koeffizienten für den Landesteil und die Erwerbstätigkeit waren nicht signifikant; Durban-Watson = 2,012, Anteil erklärter Varianz der abhängigen Variable $\left(\right.$ adj. $\mathbf{R}^{2)}=0,283$.
} 
Tab. 3 Diskriminierungserfahrungen in den Jahren von 2003 bis 2013: Situationen und Kontexte $(n=706)$ (Fortsetzung)

\begin{tabular}{|c|c|c|c|}
\hline Erfahrene ungerechtfertigte Ungleichbehandlung ${ }^{\mathrm{a}}$ & $N^{\mathrm{b}}$ & $n^{\mathrm{c}}$ & $\%^{\mathrm{d}}$ \\
\hline \multicolumn{4}{|l|}{ Kontext Schule } \\
\hline Ausschluss von Schulausflügen & 245 & 6 & 2,4 \\
\hline \multicolumn{4}{|l|}{ Kontext Erwerbstätigkeit } \\
\hline Unfreiwillige Aufgabe eines Berufsziels & 333 & 34 & 10,2 \\
\hline Verweigerung der anvisierten Berufsausbildung & 306 & 20 & 6,5 \\
\hline Ablehnung bei Stellenbewerbung & 348 & 24 & 6,9 \\
\hline Entzug einer leitenden Funktion & 286 & 13 & 4,5 \\
\hline Entzug einer leitenden Funktion wegen einer Hypoglykämie & 285 & 9 & 3,2 \\
\hline Empfehlung besonderer Arbeitszeiten & 447 & 20 & 4,5 \\
\hline Entzug interessanter beruflicher Tätigkeiten & 456 & 25 & 5,5 \\
\hline Verweigerung einer Beförderung & 442 & 19 & 4,3 \\
\hline Angst und Zurückhaltung seitens der Arbeitskolleg*innen & 480 & 75 & 15,6 \\
\hline Einmischung in Essgewohnheiten seitens der Arbeitskolleg*innen & 482 & 122 & 25,3 \\
\hline Aufforderung zur Kündigung & 438 & 13 & 3,0 \\
\hline Entlassung & 442 & 12 & 2,7 \\
\hline Entlassung und Antrag auf IV-Rente nahegelegt & 440 & 11 & 2,5 \\
\hline Aufforderung zur Frühpensionierung & 442 & 19 & 4,3 \\
\hline \multicolumn{4}{|l|}{ Kontext Militär } \\
\hline Bei der Aushebung für dienstuntauglich erklärt & 99 & 13 & 13,1 \\
\hline Bei der Aushebung für zivilschutzuntauglich erklärt & 96 & 9 & 9,4 \\
\hline Aus der Armee entlassen & 97 & 7 & 7,2 \\
\hline $\begin{array}{l}\text { Verpflichtung zu Wehrpflichtersatz trotz Bereitschaft, Militärdienst } \\
\text { zu leisten }\end{array}$ & 101 & 17 & 16,8 \\
\hline $\begin{array}{l}\text { Verpflichtung zu Wehrpflichtersatz trotz Bereitschaft, Zivilschutz zu } \\
\text { leisten }\end{array}$ & 101 & 12 & 11,9 \\
\hline \multicolumn{4}{|l|}{ Kontext Mobilität } \\
\hline $\begin{array}{l}\text { Sonderbehandlung und -prüfung beim Sicherheitscheck am Flugha- } \\
\text { fen }\end{array}$ & 463 & 81 & 17,5 \\
\hline $\begin{array}{l}\text { Auflage. ärztliche Atteste beizubringen, um die Fahrerlaubnis zu } \\
\text { erhalten }\end{array}$ & 464 & 49 & 10,6 \\
\hline \multicolumn{4}{|l|}{ Kontext Steuern } \\
\hline $\begin{array}{l}\text { Ablehnung von Abzügen von Krankheitskosten seitens des Steuer- } \\
\text { amtes }\end{array}$ & 571 & 133 & 23,3 \\
\hline \multicolumn{4}{|l|}{ Kontext Versicherungen } \\
\hline Verweigerung Lebensversicherung & 246 & 41 & 16,7 \\
\hline Verweigerung Erwerbsausfallversicherung & 249 & 19 & 7,6 \\
\hline Verweigerung Taggeldversicherung & 269 & 24 & 8,9 \\
\hline $\begin{array}{l}\text { Verweigerung Zusatzversicherung zur obligatorischen Krankenver- } \\
\text { sicherung }\end{array}$ & 301 & 73 & 24,3 \\
\hline $\begin{array}{l}\text { Kündigung Zusatzversicherung zur obligatorischen Krankenversi- } \\
\text { cherung }\end{array}$ & 463 & 15 & 3,2 \\
\hline Leistungsvorbehalt der Pensionskasse & 400 & 24 & 6,0 \\
\hline
\end{tabular}

${ }^{\mathrm{a}}$ Mehrfachantworten; ${ }^{\mathrm{b}}$ Anzahl Antwortende; ${ }^{\mathrm{c} B e f r a g t e, ~ d i e ~ a n g e b e n, ~ d i e s e ~ E r f a h r u n g ~ g e m a c h t ~ z u ~ h a b e n ; ~}$

${ }^{\mathrm{d}}$ Anteil an den Antwortenden 
Tab. 4 Diskriminierungserfahrungen im ersten Jahr nach der Diagnose: Situationen und Kontexte $(n=56)$

\begin{tabular}{|c|c|c|c|}
\hline Erfahrene ungerechtfertigte Ungleichbehandlung ${ }^{\mathrm{a}}$ & $N^{\mathrm{b}}$ & $n^{\mathrm{c}}$ & $\%^{\mathrm{d}}$ \\
\hline \multicolumn{4}{|l|}{ Kontext Freizeitgestaltung und sozialer Kontakt } \\
\hline Ungerechtfertigte Andersbehandlung im Bekanntenkreis & 51 & 5 & 9,8 \\
\hline Sich beim Essen mit Freund*innen ständig rechtfertigen müssen & 53 & 18 & 34,0 \\
\hline Verhinderung der Ausübung einer gewünschten Sportart & 41 & 5 & 12,2 \\
\hline Verweigerung des Zugangs zu Ausgehlokalen & - & - & - \\
\hline \multicolumn{4}{|l|}{ Kontext Schule } \\
\hline Ausschluss von Schulausflügen & - & - & - \\
\hline \multicolumn{4}{|l|}{ Kontext Erwerbstätigkeit } \\
\hline Unfreiwillige Aufgabe eines Berufsziels & 24 & 2 & 8,3 \\
\hline Verweigerung der avisierten Berufsausbildung & 20 & 1 & 5,0 \\
\hline Entzug interessanter beruflicher Tätigkeiten & 35 & 1 & 2,9 \\
\hline Angst und Zurückhaltung seitens Arbeitskolleg*innen & 37 & 4 & 10,8 \\
\hline Einmischung in Essgewohnheiten seitens Arbeitskolleg*innen & 38 & 9 & 23,7 \\
\hline Aufforderung zur Kündigung & 33 & 2 & 6,1 \\
\hline Entlassung & 33 & 2 & 6,1 \\
\hline Entlassung und Antrag auf IV-Rente nahegelegt & 34 & 1 & 2,9 \\
\hline Aufforderung zur Frühpensionierung & 31 & 2 & 6,5 \\
\hline \multicolumn{4}{|l|}{ Kontext Mobilität } \\
\hline Sonderbehandlung und -prüfung beim Sicherheitscheck am Flughafen & 28 & 5 & 17,9 \\
\hline \multicolumn{4}{|l|}{ Kontext Steuern } \\
\hline Ablehnung von Abzügen von Krankheitskosten seitens des Steueramtes & 33 & 3 & 9,1 \\
\hline \multicolumn{4}{|l|}{ Kontext Versicherungen } \\
\hline Verweigerung Lebensversicherung & 13 & 1 & 7,7 \\
\hline Verweigerung Zusatzversicherung zur obligatorischen Krankenversicherung & 14 & 2 & 14,3 \\
\hline
\end{tabular}

${ }^{\mathrm{a}}$ Mehrfachantworten; ${ }^{\mathrm{b}}$ Anzahl Antwortende; ${ }^{\mathrm{c} B e f r a g t e, ~ d i e ~ a n g e b e n, ~ d i e s e ~ E r f a h r u n g ~ g e m a c h t ~ z u ~ h a b e n ; ~}$

${ }^{\mathrm{d}}$ Anteil an den Antwortenden

23,2 \% der Befragten führen Erfahrungen aus einer Situation, 12,4 \% aus zwei Situationen an. Die übrigen Befragten nennen Erfahrungen in mehreren Situationen. Betrachtet man jene Befragten, die überhaupt je eine Ungleichbehandlung wahrgenommen haben $(n=408)$, liegt der Median bei Erfahrungen in zwei Situationen.

Zur Ermittlung besonders vulnerabler Gruppen wurde wiederum die Anzahl Situationen, in denen die Befragten schon eine Ungleichbehandlung wegen Diabetes erfahren haben, auf ihre Zusammenhänge mit diabetesbezogenen und anderen soziodemografischen Variablen hin analysiert. Die multiple Regressionsanalyse zeigt wiederum einen mittleren Zusammenhang zwischen der Anzahl Situationen, in denen die Befragten eine ungerechtfertigte Ungleichbehandlung erfahren haben, und ihrem Alter $(\beta=-0,43 ; p \leq 0,001)$ sowie ihrer Lebenszeit mit Diabetes auf $(\beta=$ $-0,14 ; p \leq 0,001)^{2}$. Betrachtet man die Angaben derjenigen Befragten, die erst ein

\footnotetext{
2 Weitere Angaben zum Regressionsmodell: $N=672$; die standardisierten Regressionskoeffizienten $\beta$ für den Diabetestyp, das Geschlecht, den Landesteil und die Erwerbstätigkeit waren nicht signifikant; DurbanWatson $=1,926$, Anteil erklärter Varianz der abhängigen Variable (adj. $\left.R^{2}\right)=0,156$.
} 
Jahr vor der Befragung Diabetes diagnostiziert erhalten haben $(n=56)$, erhält man einen Einblick in die Erfahrungen, die sich im Jahr 2013 bereits in den ersten Monaten nach der Diagnose einstellten. $49 \%$ der Befragten, die zum Zeitpunkt der Befragung nicht mehr als ein Jahr mit Diabetes gelebt haben, geben an, dass sie bislang keine der aufgeführten Situationen ungerechtfertigter Ungleichbehandlung erlebt haben. $51 \%$ der Befragten gibt hingegen an, dass sie wegen Diabetes schon ungerechtfertigt ungleich behandelt worden sind. Dabei zeigt sich, dass diese Befragten, die überhaupt nur auf Erfahrungen in diesem Zeitraum zurückblicken können, in 16 von 33 Situationen Erfahrungen von ungerechtfertigter Ungleichbehandlung gemacht haben (vgl. Tab. 4).

\subsection{Wahrgenommene Zuschreibungen}

Die Befragten nehmen wahr, welche stereotypen Zuschreibungen mit dem Label „Diabetiker“ verbunden sind und in der Interaktion mit Dritten zum Ausdruck kommen. Die Angaben der 3347 Befragten bestätigen zunächst, dass die teilnehmenden Menschen mit Diabetes alle aufgeführten Zuschreibungen schon einmal wahrgenommen haben. Wie die Darstellung der absoluten Anzahl Nennungen veranschaulicht (Tab. 5), gibt es keine Zuschreibung, die ohne Nennung geblieben wäre. Nur 15,6 \% der Befragten $(n=516)$ gaben an, bislang keine der genannten stereotypen Zuschreibungen wahrgenommen zu haben. Bei den übrigen ist die Spannweite beträchtlich und reicht von der Wahrnehmung einer Zuschreibung bis hin zum Bewusstsein um 26 Zuschreibungen.

\subsection{Muster der Zuschreibungen}

Um die Muster der wahrgenommenen Stereotype zu ermitteln, wurden die Antworten einer Faktorenanalyse unterzogen. Die Analyse ergab eine Lösung mit fünf Faktoren. Dieses Ergebnis kann (unter Berücksichtigung der Ladungen über dem cut off von 0,30) sinnvoll als fünf inhaltlich unterschiedliche Profile von stereotypen Zuschreibungen interpretiert werden:

Die fünf Profile (in Tab. 6 kursiv) können als inhaltliche Dimensionen der stereotypen Zuschreibungen verstanden werden, die jeweils eine bestimmte Stoßrichtung zeigen und die Personen mit Diabetes in bestimmter Form objektivieren. Sie stellen Menschen mit Diabetes also als bestimmte Charaktere dar.

Das erste Profil umfasst Stereotypen, die Menschen mit Diabetes eine Behinderung zuschreiben, Defizite unterstellen und sie als fremd und nicht dazugehörig stilisieren. Menschen mit Diabetes werden von diesen Zuschreibungen diskursiv als „sonderliche Minderwertige“ objektiviert. Das zweite Profil bündelt mitleidsgetragene, dramatisierende und paternalistische, entmündigende Zuschreibungen. Menschen mit Diabetes werden als ,arme Kranke“ objektiviert, die eine Sonderbehandlung brauchen. Das dritte Profil charakterisiert Menschen mit Diabetes als willensschwache, gefrässige, übergewichtige und alte Personen, die mit ihrem Verhalten ihre Krankheit selbst verschuldet haben und die Steuerzahler belasten. Diabetiker*innen werden damit als ,haltlose Unverantwortliche“ dargestellt. Das vierte Profil kreist um die Zuschreibung von Leistungsschwäche und zeigt einen deutlichen Zusam- 
Tab. 5 Wahrgenommene Stereotype $(N=3347)$

\begin{tabular}{|c|c|c|c|}
\hline Wahrgenommene stereotype Zuschreibung ${ }^{\mathrm{a}}$ & $N^{\mathrm{b}}$ & $\mathrm{n}^{\mathrm{c}}$ & $\%^{\mathrm{d}}$ \\
\hline \multicolumn{4}{|l|}{ Ich hatte schon einmal das Gefühl, dass ... } \\
\hline ... Leute denken, Menschen mit Diabetes seien ,arme Kranke“ & 3234 & 1227 & 37,9 \\
\hline ... Leute denken, Menschen mit Diabetes seien bemitleidenswert & 3222 & 1216 & 37,7 \\
\hline ... Leute denken, Diabetes sei etwas Schreckliches & 3206 & 1370 & 42,7 \\
\hline $\begin{array}{l}\text {... Freund*innen oder Bekannte denken, Menschen mit Diabetes brauch- } \\
\text { ten eine Spezialbehandlung }\end{array}$ & 3190 & 1119 & 35,1 \\
\hline $\begin{array}{l}\text {... Leute denken, Menschen mit Diabetes würden ihre Krankheit als } \\
\text { Vorwand einsetzen, um davon zu profitieren }\end{array}$ & 3208 & 402 & 12,5 \\
\hline ... Leute Menschen mit Diabetes für Simulanten halten & 3219 & 360 & 11,2 \\
\hline $\begin{array}{l}\text {... Leute denken, Menschen mit Diabetes würden oft krankheitsbedingt } \\
\text { ausfallen }\end{array}$ & 3184 & 641 & 20,1 \\
\hline $\begin{array}{l}\text {... Leute denken, Menschen mit Diabetes seien in ihrer Leistung beein- } \\
\text { trächtigt }\end{array}$ & 3196 & 1289 & 40,3 \\
\hline ... Leute denken, Menschen mit Diabetes nicht belastbar seien & 3180 & 1002 & 31,5 \\
\hline $\begin{array}{l}\ldots \text { Leute denken, Menschen mit Diabetes schädigten das Image der Orga- } \\
\text { nisation/Firma, in der sie arbeiten }\end{array}$ & 3128 & 120 & 3,8 \\
\hline ... Leute denken, Menschen mit Diabetes seien invalid & 3180 & 357 & 11,2 \\
\hline ... Leute denken, Menschen mit Diabetes hätten ein Defizit & 3160 & 721 & 22,8 \\
\hline ... Leute denken, Menschen mit Diabetes seien behindert & 3171 & 574 & 18,1 \\
\hline ... Leute denken, Menschen mit Diabetes seien ein Risikofaktor & 3167 & 1208 & 38,1 \\
\hline $\begin{array}{l}\text {... Leute denken, Menschen mit Diabetes würden den Steuerzahler mit } \\
\text { ihren Gesundheitskosten belasten }\end{array}$ & 3188 & 903 & 28,3 \\
\hline ... Leute Menschen mit Diabetes für Exoten halten & 3170 & 264 & 8,3 \\
\hline $\begin{array}{l}\text {... Freunde oder Bekannte denken, Menschen mit Diabetes gehörten } \\
\text { nicht zu ihnen }\end{array}$ & 3185 & 124 & 3,9 \\
\hline ... Leute Diabetiker*innen fälschlicherweise als Junkies betrachtet haben & 3157 & 475 & 15 \\
\hline $\begin{array}{l}\text {... Leute Diabetiker*innen, die in der Öffentlichkeit Insulin spritzen, } \\
\text { komisch anschauen }\end{array}$ & 3127 & 1726 & 55,2 \\
\hline ... Leute denken, Menschen mit Diabetes seien weniger wert & 3172 & 333 & 10,5 \\
\hline ... Leute denken, Menschen mit Diabetes seien faul & 3172 & 280 & 8,8 \\
\hline ... Leute denken, Menschen mit Diabetes seien alt und übergewichtig & 3191 & 1526 & 47,8 \\
\hline ... Leute denken, Menschen mit Diabetes wären gefräßig & 3174 & 717 & 22,5 \\
\hline ... Leute denken, Menschen mit Diabetes seien willensschwach & 3189 & 544 & 17,1 \\
\hline ... Leute denken, Diabetes sei selbst verschuldet & 3192 & 1260 & 39,5 \\
\hline $\begin{array}{l}\text {... Menschen mit Diabetes nur noch als Diabeteskranke wahrgenommen } \\
\text { werden }\end{array}$ & 3172 & 875 & 27,6 \\
\hline
\end{tabular}

${ }^{\mathrm{a}}$ Mehrfachantworten; ${ }^{\mathrm{b}}$ Anzahl Antwortende; ${ }^{\mathrm{c} B e f r a g t e, ~ d i e ~ a n g e b e n, ~ d i e s e ~ Z u s c h r e i b u n g ~ w a h r g e n o m m e n ~}$ zu haben; ${ }^{\mathrm{d}}$ Anteil an den Antwortenden

menhang zum Kontext Erwerbstätigkeit. Menschen mit Diabetes werden von diesen Zuschreibungen als „Leistungsschwache“ objektiviert. Das fünfte Profil stellt Menschen mit Diabetes als Simulant*innen dar, die ihre Krankheit zu ihren Gunsten nutzen. Diese Zuschreibungen objektivieren Diabetiker*innen als „Profiteure“. Die übrigen vier Zuschreibungen sind schwach $(\leq 0,30)$, laden auf mehrere Profile und erweisen sich damit für keines spezifisch. 
Tab. 6 Faktorenanalyse der wahrgenommenen Stereotype

\begin{tabular}{|c|c|c|c|c|c|}
\hline \multirow{2}{*}{$\begin{array}{l}\text { Stereotype } \\
\text { Menschen mit Diabetes ... }\end{array}$} & \multicolumn{5}{|c|}{ Faktoren } \\
\hline & 1 & 2 & 3 & 4 & 5 \\
\hline ... sind invalid & 0,672 & $-0,078$ & 0,014 & $-0,020$ & 0,048 \\
\hline ... sind behindert & 0,600 & $-0,103$ & 0,042 & $-0,119$ & 0,126 \\
\hline ... haben ein Defizit & 0,507 & $-0,106$ & 0,008 & $-0,196$ & 0,087 \\
\hline ... sind weniger wert & 0,499 & 0,048 & $-0,134$ & $-0,065$ & $-0,056$ \\
\hline ... gehören nicht dazu & 0,465 & 0,018 & $-0,034$ & 0,096 & $-0,146$ \\
\hline ... schädigen das Image der Organisation & 0,405 & 0,031 & 0,013 & 0,005 & $-0,143$ \\
\hline ... sind Exoten & 0,363 & $-0,089$ & $-0,088$ & 0,054 & $-0,095$ \\
\hline $\begin{array}{l}\text {... werden nur noch als Diabeteskranke } \\
\text { wahrgenommen }\end{array}$ & 0,229 & 0,101 & $-0,135$ & $-0,205$ & $-0,047$ \\
\hline ... sind bemitleidenswert & $-0,016$ & $-0,828$ & $-0,008$ & 0,085 & $-0,006$ \\
\hline ... sind arme Kranke & 0,023 & $-0,761$ & $-0,009$ & 0,024 & $-0,063$ \\
\hline ... haben eine schreckliche Krankheit & 0,027 & $-0,509$ & $-0,055$ & $-0,078$ & $-0,019$ \\
\hline ... brauchen eine Spezialbehandlung & 0,143 & $-0,360$ & $-0,015$ & $-0,160$ & 0,044 \\
\hline $\begin{array}{l}\text {... werden beim Spritzen komisch ange- } \\
\text { schaut }\end{array}$ & $-0,013$ & $-0,230$ & $-0,195$ & $-0,199$ & $-0,023$ \\
\hline ... werden als Junkies betrachtet & 0,145 & $-0,189$ & $-0,070$ & $-0,031$ & $-0,170$ \\
\hline ... sind gefrässig & $-0,036$ & $-0,035$ & $-0,780$ & 0,099 & 0,001 \\
\hline ... sind willensschwach & 0,140 & 0,042 & $-0,635$ & 0,067 & $-0,070$ \\
\hline ... sind alt und übergewichtig & $-0,060$ & $-0,049$ & $-0,622$ & $-0,118$ & 0,076 \\
\hline ... haben Diabetes selbst verschuldet & $-0,023$ & $-0,077$ & $-0,595$ & $-0,105$ & $-0,006$ \\
\hline $\begin{array}{l}\text {... belasten Steuerzahler mit ihren Ge- } \\
\text { sundheitskosten }\end{array}$ & 0,136 & 0,024 & $-0,318$ & $-0,188$ & $-0,104$ \\
\hline ... sind in ihrer Leistung beeinträchtigt & 0,024 & $-0,078$ & $-0,023$ & $-0,706$ & $-0,052$ \\
\hline ... sind nicht belastbar & 0,053 & $-0,036$ & $-0,076$ & $-0,622$ & $-0,088$ \\
\hline ... fallen oft krankheitsbedingt aus & 0,051 & $-0,069$ & $-0,026$ & $-0,433$ & $-0,282$ \\
\hline ... sind ein Risikofaktor & 0,125 & $-0,078$ & $-0,104$ & $-0,363$ & $-0,071$ \\
\hline $\begin{array}{l}\text {... nutzen Diabetes als Vorwand, um zu } \\
\text { profitieren }\end{array}$ & $-0,026$ & $-0,076$ & 0,027 & $-0,126$ & $-0,707$ \\
\hline ... sind Simulanten & 0,060 & $-0,031$ & $-0,045$ & $-0,048$ & $-0,701$ \\
\hline ... sind faul & 0,282 & 0,073 & $-0,268$ & 0,023 & $-0,290$ \\
\hline
\end{tabular}

$N=3347$; Extraktionsmethode: Maximum Likelihood; Rotationsmethode: Oblimin mit Kaiser Normalisierung; Rotation konvergierte in 14 Iterationen; Bartlett's Test der Sphärizität: $p<0,001$; Kaiser-MeyerOlkin Test $=0,934$; erklärter Anteil Varianz $=41,06$

\section{Diskussion}

Die vorliegende Untersuchung zeigt, dass Menschen mit Diabetes in der Schweiz in verschiedenen Situationen und Kontexten die Erfahrung machen, wegen ihres Diabetes ungerechtfertigt ungleich behandelt zu werden. Mag die öffentliche Einschätzung auch dahin gehen, Diabetes sei eine Krankheit, die kaum von Stigmatisierung betroffen sei (Fernandes et al. 2007), zeigt die hier dargelegte Untersuchung, dass Menschen mit Diabetes in der Schweiz durchaus Stigmatisierung erfahren. Ein knappes Drittel der Befragten gab an, sie seien noch nie wegen Diabetes diskriminiert worden. 68,5\% der Befragten gaben an, solche Erfahrungen gemacht 
zu haben - auch in mehreren Situationen. Die Hälfte derjenigen, die schon ungerechtfertigte Ungleichbehandlung erlebt haben, nennen bis zu drei Situationen. Fokussiert man auf die Erfahrungen jener, deren Diagnose im Jahr 2003 oder später erfolgte, ergibt sich eine ähnliche Feststellung. In dieser Gruppe zeigt sich, dass $59 \%$ der Befragten in einer oder mehreren Situationen die Erfahrung gemacht haben, wegen Diabetes ungerechtfertigt ungleichbehandelt worden zu sein. Betrachtet man jene, die maximal ein Jahr mit der Diagnose lebten, zeigt sich, dass $51 \%$ dieser kleinen Gruppe bereits kurze Zeit nach ihrer Diagnose Erfahrungen von ungerechtfertigter Ungleichbehandlung gemacht haben. Damit wird deutlich, dass die hier erfassten Diskriminierungserfahrungen durchaus eine gewisse Aktualität haben. Kann bei der Betrachtung der Lebenszeitprävalenz nicht danach unterschieden werden, ob weit zurückliegende Erfahrungen geschildert werden oder erst kürzlich gemachte, so kann aus der Analyse der Angaben ausschließlich jener Befragten, die höchstens zehn bzw. ein Jahr mit Diabetes leben, geschlossen werden, dass die Erfahrungen im Zeitraum nach 2003 gemacht wurden. Die Erfahrungen, von denen sie berichten, können damit nicht einfach als Einblicke in längst vergangene Zeiten und überwundene Verhältnisse abgetan werden. Die Angaben zur Auftretenshäufigkeit der einzelnen Erfahrungen im Zeitraum von 2003 bis 2013 bzw. im Jahr vor der Befragung dürften aufgrund des hier gewählten Vorgehens allerdings systematisch zu gering ausfallen, denn all jene Befragte, die schon länger als zehn Jahre bzw. ein Jahr mit Diabetes lebten, wurden in der entsprechenden Analyse nicht berücksichtigt. Ihre Erfahrungen, die sie im selben Zeitraum gemacht haben mögen, treten damit nicht in den Blick. Der Vorteil dieses konservativen Vorgehens liegt darin, dass es Fehlangaben aufgrund ungenauer Erinnerung ausschliesst, da mit Sicherheit keine diabetesbedingten Diskriminierungserfahrungen genannt werden können, die länger zurückliegen als die gewählte Zeitspanne von zehn Jahren bzw. einem Jahr. Aufgrund des jungen Forschungsstands können nur einige wenige der Erfahrungen, die hier erfasst wurden, mit Ergebnissen anderer Studien sinnvoll verglichen und damit extern validiert werden. Dies ist nur mit Blick auf die Untersuchung zu Diskriminierungen im Kontext der Erwerbsarbeit in der Schweiz möglich. Pietra Nebiker-Pedrotti und Kolleg*innen (2009) kamen zum Ergebnis, dass 5 \% der untersuchten Menschen mit Diabetes angaben, sie hätten schon (mindestens) einmal wegen Diabetes ihre Anstellung verloren. $10 \%$ hätten schon einmal die Erfahrung gemacht, dass ihre Bewerbung wegen ihrer Krankheit abgelehnt wurde. Bis zu $15 \%$ der Befragten berichteten von Problemen mit Versicherungen (Nebiker-Pedrotti et al. 2009). In unserer Untersuchung gaben 2,6 \% der Befragten an, schon einmal wegen Diabetes die Stelle verloren zu haben und weitere $3 \%$ erklärten, dass ihnen gekündigt und gleichzeitig nahegelegt worden war, sich bei der Invalidenversicherung abhängig zu machen. Mit total 5,6 \% ist dieser Anteil der Befragten mit den Zahlen vergleichbar, die von Nebiker-Pedrotti und Kolleg*innen (2009) berichtet wurden. Es sind allerdings auch weniger offensichtliche Formen von Stellenverlust oder -gefährdung zu verzeichnen: Nach unserer Untersuchung wurden 4,8\% der Befragten schon aufgefordert, sich frühzeitig pensionieren zu lassen. Und weitere 3,3\% der Befragten gaben an, wegen Diabetes schon einmal aufgefordert worden zu sein, selbst zu kündigen. Alle diese Erfahrungen können in den Verlust einer Anstellung münden. Sie entsprechen den Erfahrungen von 13,7 \% 
der Befragten. Der Anteil der Befragten, die wegen Diabetes schon einmal eine Stelle nicht erhalten haben, beläuft sich auf $8 \%$ und liegt damit leicht unterhalb des Anteils, der bisher referiert wurde (Nebiker-Pedrotti et al. 2009). Weiter lassen sich die Erfahrungen mit dem Abschluss einer Taggeldversicherung vergleichen. In unserer Befragung vermerkten 13,2 \% der Befragten, die sich nach ihrer Diabetesdiagnose um eine Taggeldversicherung bemüht hatten, dass ihnen eine solche verweigert wurde. Bei Nebiker-Pedrotti liegt der analoge Wert bei 6\% der Befragten. Fasst man weitere Erfahrungen mit Versicherungen in den Blick, so wird deutlich, dass es nicht Taggeldversicherungen sind, die am häufigsten zu Problemen führen. Nach unserer Befragung haben 26,4 \% der Befragten die Erfahrung gemacht, dass ihnen eine Zusatzversicherung zur obligatorischen Krankenversicherung verweigert wurde, während gar 28,6 \% keine Lebensversicherung abschliessen konnten.

Ein Blick auf die Angaben der Befragten zeigt, dass Ungleichbehandlung in alltäglichen Umgang mit Kolleg*innen an der Arbeit, aber auch im Freizeitbereich häufig sind. Weiter lässt sich festhalten, dass dabei bezeichnenderweise Situationen im Vordergrund stehen, in denen die Befragten durch strukturelle Barrieren oder strukturell mit Macht ausgestattete Entscheidungsträger in der Realisierung ihrer Verwirklichungschancen beeinträchtigt werden. Ihre Wahrnehmung konzentriert sich auf Effekte von Gesetzen, Regelungen, Administrations- und Behördenhandeln oder Entscheidungen von Arbeitgebern. Hierin bestätigt sich die theoretische Überlegung von Link und Phelan (2001), dass Stigmatisierungsprozesse eng an Machtausübung gekoppelt sind. Die Studie zeigt auch auf, welche stereotypen Zuschreibungen die Befragten bisher schon wahrgenommen haben. Die Palette der Stereotypen ist reich abschattiert und reicht von Mitleidsäusserungen bis hin zu verachtenden und erniedrigenden Aussagen und Unterstellungen. Die hier präsentierten Ergebnisse bestätigen den Befund früherer Untersuchungen, dass in der Öffentlichkeit abwertende Bilder über Menschen mit Diabetes bestehen. Diese Ergebnisse sind differenzierter als jene einer in der New York City durchgeführten Studie, die bei Passanten das Bild von Personen mit Diabetes Typ 1 erhob, bestätigen aber die dort erfassten Zuschreibungen, Menschen mit Diabetes seien selbst für ihre Krankheit verantwortlich, seien faul, übergewichtig und hätten ungesunde Essgewohnheiten (Vishwanath 2014). Der Vorwurf, die Betroffenen würden das Gesundheitswesen unnötig finanziell belasten, wie auch die Verwechslung mit Drogenabhängigen bestätigen Aussagen anderer kürzlich erschienener Studien (Browne et al. 2013; Schabert et al. 2013).

Wie jede Studie hat auch diese gewisse Einschränkungen. Die Erfassung von Ungleichbehandlung stellt allein auf Angaben der davon betroffenen Menschen mit Diabetes ab, was Verzerrungen zur Folge haben kann. Die Diskriminierungsforschung zeigt, dass die Sensibilität der Wahrnehmung von Diskriminierung von sozialstrukturellen, individuellen und situativen Faktoren beeinflusst wird (El-Mafaalani et al. 2017). Dies ist allerdings nicht mit der Aussage zu verwechseln, die angeführten Beispiele von ungerechtfertigter Ungleichbehandlung hätten nicht wirklich stattgefunden. Deshalb kann auch mit den Worten von Aladin El-Mafaalani und Kolleg*innen (2017) festhalten werden, dass die Betroffenenperspektive ,sowohl für die Diskriminierungsforschung als auch für die pädagogische Praxis sowie die politische und öffentliche Auseinandersetzung mit Diskriminierung und Anti-Diskriminerungsstrategien von zentraler Relevanz (ist) - nicht zuletzt auch deshalb, weil 
Problembewusstsein und Eigeninitiative der Betroffenen für die Entwicklung von Gegenmaßnahmen von entscheidender Bedeutung sind“ (El-Mafaalani et al. 2017, S. 174). Die retrospektive Erfassung über die ganze bisherige Lebenszeit bzw. zehn Jahre kann dazu führen, dass weniger massive Formen der Ungleichbehandlung dem Vergessen geschuldet nicht angegeben und in der Konsequenz nicht sichtbar werden. Zudem war die Studie gezwungen, mit einem convenience sample zu arbeiten. Die Rücklaufquote war gering, auch wenn sie in einem Bereich lag, der für Befragungen ohne Mahnungsmöglichkeit üblich ist (Diekmann 1995). Die Art der Stichprobe wie auch die Rücklaufquote könnten zu einer Verzerrung der Ergebnisse geführt haben. Ein Vergleich mit den jüngsten epidemiologischen Schätzungen (Huber et al. 2014) zeigt immerhin, dass die Geschlechterverteilung im Sample dieser Studie (45,2 \% Frauen und 54,8 \% Männer) sehr nahe an die Geschlechterverteilung im Sample der Studie herankommt, auf der die Schätzung der Prävalenz von Diabetes in der Schweiz von 2011 abstellt (45,7 \% Frauen und 54,3 \% Männer). Auch die Altersverteilungen liegen nahe beieinander. In der Stichprobe dieser Untersuchung waren $70,9 \%$ der Befragten älter als 56 Jahre während in der Studie zu Prävalenzschätzung 73,7 \% über 59 Jahre alt waren (Huber et al. 2014). In dieser Studie dürfte allerdings der Anteil junger Menschen mit Diabetes und Menschen mit Diabetes Typ 1 übervertreten sein.

Angesichts des geringen gesicherten Wissens über Stigmatisierung von Menschen mit Diabetes bringt die vorliegende Studie dennoch eine wichtige Erweiterung der Erkenntnisse. Sie erfasst Erfahrungen von ungerechtfertigter Ungleichbehandlung in einem breiten Spektrum von Lebenskontexten und vermag zu umreissen, wie groß der Anteil der davon betroffenen Menschen mit Diabetes ist. Zudem macht sie die Vielfalt und Muster von stereotypen Zuschreibungen fassbar, denen sich Menschen mit Diabetes gegenübersehen. Wie die International Diabetes Federation fordert, bedarf die in dieser Studie porträtierte Situation der Veränderung (Benedetti 2014). Die Zeiten, in denen Menschen mit Diabetes dazu neigten, Stigmatisierung ,passiv zu akzeptieren“ (Wardian und Sun 2014), scheinen allerdings vorüber. Die Ergebnisse dieser Befragung lassen erkennen, dass die jüngere Generation von Menschen mit Diabetes sich häufiger von Stigmatisierung betroffen sehen. Sie gaben häufiger an, ungerechtfertigte Ungleichbehandlung erfahren und Stereotype wahrgenommen zu haben, als die ältere Generation in der Stichprobe. Dieses Ergebnis könnte als Ausdruck vermehrter Ungleichbehandlung von jüngeren Menschen mit Diabetes verstanden werden. Angesichts der stärkeren Eingebundenheit in die Arbeitswelt kann diese Sicht eine gewisse Plausibilität in Anspruch nehmen. Das Ergebnis kann aber auch dahingehend interpretiert werden, dass Angehörige dieser Generation Diskriminierung gegenüber sensibler sind, ungerechtfertigte Ungleichbehandlung nicht als unvermeidbare Begleiterscheinung ihres Gesundheitszustands zu betrachten bereit sind, Diskriminierung nicht normalisieren oder stereotype Zuschreibungen internalisieren und als quasi natürlicher Teil ihrer chronischen Krankheit verstehen. Dies würde ein Potenzial markieren, Ungleichbehandlung anzugehen. Und dies ist für Menschen mit Diabetes eine gute Ausgangslage für den Kampf für Chancengleichheit und gegen Stigmatisierung, gegen Ungleichbehandlung, Benachteiligung und Ausschluss. 
Hierzu sind Maßnahmen zu diskutieren, zu entwickeln und einzuleiten. Ein naheliegender Weg zur Bearbeitung der Ungleichbehandlung führt über die Klage gegen Diskriminierung. Diese Strategie hat hinsichtlich der Behandlung durch die Schweizer Armee Früchte getragen. Die oft beschriebene Situation, dass jemand wegen Diabetes gegen den eigenen Willen für dienstuntauglich erklärt wird und dennoch Militärersatzsteuer bezahlen muss, ist erfolgreich über den Rechtsweg angegangen worden. Ein Betroffener hat am Europäischen Gerichtshof für Menschenrechte dagegen geklagt. Mit Spruch vom 30. April 2009 rügte das Gericht unter anderem, dass eine andere Praxis denkbar wäre, als Stellungspflichtige mit Diabetes systematisch für untauglich zu erklären (European Court of Human Rights 2009). Angesichts des in der Schweiz schwach entwickelten Diskriminierungsschutzes, sind die Möglichkeiten, auf juristischem Weg etwas gegen Ungleichbehandlung ausrichten, stark begrenzt. Wie ein juristisches Gutachten aufzeigt, würden Erfolge vor Gericht voraussetzen, dass sich Menschen mit Diabetes als „Behinderte“ definierten (Pärli 2009). Wie die qualitative Studie erkennen lässt, ist die Selbstdefinition als ,Behinderte“ umstritten, für einige Betroffene inakzeptabel (Raemy et al. 2012, S. 41). Es scheint deshalb angezeigt, weitere Strategien zu entwerfen, mit denen gegen manifeste Diskriminierung vorgegangen und die stereotypen Zuschreibungen aufgegriffen und zugunsten von differenzierten Vorstellungen über Menschen mit Diabetes zurückgewiesen werden können.

\section{Implikationen für die Soziale Arbeit}

Die vorgelegten Ergebnisse scheinen uns nicht ohne Konsequenzen für die Soziale Arbeit zu bleiben: Sozialarbeitende, die mit Menschen mit Diabetes arbeiten, sind in einer guten Position, sich im einzelnen Fall für konkret Betroffene einzusetzen, Chancengleichheit einzufordern oder auch personenbezogene und fallangemessene Ungleichheit auszuhandeln. Angesichts der hier dargestellten Erkenntnisse sollte die Reichweite der Angebote Sozialer Arbeit für Menschen mit Diabetes jedoch über den einzelnen Fall hinausgehen, sich aus einer Engführung auf die Beratung und Unterstützung einzelner Betroffener und ihrer Partner*innen und Angehörigen bzw. Eltern hinaus entwickeln und die Fixierung auf die Bestärkung des Krankheitsmanagements und die Vermeidung von Folgekrankheiten - wie dies in der Literatur sonst skizziert wird (vgl. z. B. Ayalon et al. 2008) - bewusst hinter sich lassen. Angesichts der für die Soziale Arbeit typischen Perspektive, Menschen in ihrer Beziehung zu ihrer Umwelt zu sehen, sollte Soziale Arbeit auch die Interaktion von Menschen mit Diabetes mit ihrem sozialen Umfeld mit in den Blick fassen und auch auf die sozialen Reaktionen fokussieren, mit denen Menschen mit Diabetes konfrontiert sind. Stigmatisierungs- und Diskriminierungserfahrungen können in der Arbeit mit Betroffenen angesprochen und aufgearbeitet werden. Die Bewältigungsformen können thematisiert, auf ihre Folgen für die Menschen mit Diabetes selbst hinterfragt und allenfalls alternative Umfangsformen mit Stigmatisierung und Diskriminierung entwickelt und bestärkt werden.

In diesem Zusammenhang sehen wir Soziale Arbeit herausgefordert, Menschen mit Diabetes in ihrem Kampf gegen Stigmatisierung und Diskriminierung zu unter- 
stützen. Sozialarbeitende sollten bewusst einen Beitrag zur Veränderung der Wahrnehmung und Behandlung von Menschen mit Diabetes leisten. Hierfür sind allerdings erst spezifische und angemessene Vorgehensweisen $\mathrm{zu}$ entwickeln und $\mathrm{zu}$ evaluieren. Die Entwicklungsarbeit (Gredig 2011; Fraser et al. 2009) kann dabei auf den Erfahrungen im Kampf gegen die Stigmatisierung von Menschen mit HIV/Aids aufbauen (Grossman et al. 2013). Wobei sich absehen lässt, dass eine der großen Herausforderungen darin bestehen wird, die Aktivitäten zur Prävention von Diabetes Typ 2 mit den Interventionen zur Veränderung des öffentlichen Bildes von Menschen mit Diabetes vereinbar zu gestalten. Wir sehen Soziale Arbeit aufgefordert, hierzu mit Menschen mit Diabetes und ihren (Selbsthilfe-)Organisationen zusammenzuarbeiten und mit ihnen gemeinsam Strategien zur Bekämpfung von Stigmatisierung $\mathrm{zu}$ diskutieren, anzupassen, neu zu entwickeln, zu vereinbaren und schließlich zu implementieren. Die hier präsentierten Forschungsergebnisse können den Sozialarbeitenden eine Grundlage bieten. Die präsentierten Forschungsergebnisse sind aber auch für jene Sozialarbeitenden von Belang, die nicht in die Beratung von Menschen mit Diabetes einbezogen sind und nicht bewusst mit Menschen arbeiten, die mit Diabetes leben. Ob sie sich dessen bewusst werden oder nicht, treffen Sozialarbeitende in allen Handlungsfeldern mit hoher Wahrscheinlichkeit auch auf Kinder, Jugendliche und Erwachsene, die mit Diabetes leben. Die chronische Krankheit mag längst nicht in jedem professionellen Kontakt von den Betroffenen offengelegt werden. Sie muss für das primäre Ziel, das die Klient*innen mit den Sozialarbeitenden anstreben, auch nicht von Belang sein. Dessen ungeachtet sollten Sozialarbeitende aber informiert sein und ihre Bilder und ihre Haltung gegenüber Menschen mit Diabetes insoweit reflektiert haben, dass sie in ihren Interkationen mit Klient*innen - sei die Krankheit nun explizit Thema oder nicht - keine Stereotypen über Menschen mit Diabetes reproduzieren oder diskriminierend handeln.

\footnotetext{
Danksagung Wir danken den Befragten für ihre Bereitschaft, an dieser Untersuchung mitzuwirken und uns ihre Angaben zur Verfügung zu stellen. Genauso danken wir der Schweizerischen Diabetes-Gesellschaft für die verlässliche und erfreuliche Zusammenarbeit über die gesamte Laufzeit des Projekts. Weiter bedanken wir uns bei Stéphanie Grillet, die an der qualitativen Untersuchung mitwirkte, und den wissenschaftlichen Hilfskräften, die die Datenverarbeitung auch während der Saison der Feiertage engagiert vorangetrieben haben: Manon Morgenthaler, Sophie Schärer, David Fricker sowie Severin, David und Jan Hürzeler. Dr. Theo Hutter und seiner Kritik verdanken wir Anregungen zu weiteren Analysen. Nicht zuletzt gilt unser Dank dem Schweizerischen Nationalfonds wie auch der Schweizerischen Diabetes-Stiftung. Sie haben diese Untersuchung finanziell gefördert.
}

Open Access Dieser Artikel wird unter der Creative Commons Namensnennung 4.0 International Lizenz (http://creativecommons.org/licenses/by/4.0/deed.de) veröffentlicht, welche die Nutzung, Vervielfältigung, Bearbeitung, Verbreitung und Wiedergabe in jeglichem Medium und Format erlaubt, sofern Sie den/die ursprünglichen Autor(en) und die Quelle ordnungsgemäß nennen, einen Link zur Creative Commons Lizenz beifügen und angeben, ob Änderungen vorgenommen wurden.

Förderung Die Untersuchung, auf die sich dieser Beitrag bezieht, ist vom Schweizerischen Nationalfonds (Projekt Nr. 13DPD3_132288) und der Schweizerischen Diabetes-Stiftung gefördert worden. 


\section{Literatur}

Ayalon, L., Gross, R., Tabenkin, H., Porath, A., Heymann, A., \& Porter, B. (2008). Determinants of quality of life in primary care patients with diabetes: Implications for social workers. Health \& Social Work, 33(3), 229-236.

Balfe, M., Doyle, F., Smith, D., Sreenan, S., Brugha, R., Hevey, D., \& Conroy, R. (2013). What's distressing about having type 1 diabetes? A qualitative study of young adults' perspectives. BMC Endocrine Disorders. https://doi.org/10.1186/1472-6823-13-25.

Benedetti, M. M. (2014). Discrimination and diabetes. Diabetes Research and Clinical Practice, 103(2), 338-340. https://doi.org/10.1016/j.diabres.2014.02.001.

Broom, D., \& Whittaker, A. (2004). Controlling diabetes, controlling diabetics: moral language in the management of diabetes type 2. Social Science \& Medicine, 58(11), 2371-2382. https://doi.org/10. 1016/j.socscimed.2003.09.002.

Browne, J.L., Ventura, A., Mosely, K., \& Speight, J. (2013). I call it the blame and the shame disease: a qualitative study about perceptions of social stigma surrounding type 2 diabetes. BMJ Open, 3, 1-10.

Ciporen, H. (2012). Social workers' role in combating the new epidemic of type 2 diabetes in children: clinical interventions at the hall family center for pediatric endocrinology and diabetes. Social Work in Health Care, 51(1), 22-35. https://doi.org/10.1080/00981389.2011.622634.

Cohen, J. (1992). A power primer. Psychological Bulletin, 112(1), 155-159. https://doi.org/10.1037/00332909.112.1.155.

Creswell, J.W. (2009). Research design. Qualitative, quantitative, and mixed methods approaches. Los Angeles, London, New Delhi, Singapore: SAGE.

DeCoster, V. A. (2001). Challenges of type 2 diabetes and role of health care social work: a neglected area of practice. Health \& Social Work, 26(1), 26-37. https://doi.org/10.1093/hsw/26.1.26.

DeCoster, V.A., \& Cummings, S.M. (2005). Helping adults with diabetes: a review of evidence-based interventions. Health \& Social Work, 30(3), 259-264. https://doi.org/10.1093/hsw/30.3.259.

Diamantopoulos, A., \& Siguaw, J. A. (2006). Formative versus reflective indicators in organizational measure development: a comparison and empirical illustration. British Journal of Management, 17(4), 263-282. https://doi.org/10.1111/j.1467-8551.2006.00500.x.

Diekmann, A. (1995). Empirische Sozialforschung. Grundlagen, Methoden, Anwendungen. Reinbek b. Hamburg: Rowohlt.

Dillman, D. (1978). Mail and telepone surveys: the total design method. New York: John Wiley \& Sons.

Egede, L.E., \& Dismuke, C.E. (2012). Serious psychological distress and diabetes: a review of the literature. Current Psychiatry Reports, 14(1), 15-22. https://doi.org/10.1007/s11920-011-0240-0.

El-Mafaalani, A., Waleciak, J., \& Weitzel, G. (2017). Tatsächliche, messbrae und subjektiv wahrgenommene Diskriminierung. In A. Scherr, A. El-Mafaalani \& G. Yüksel (Hrsg.), Handbuch Diskriminierung (S. 173-189). Wiesbaden: Springer VS.

European Court of Human Rights (2009). Information Note on the Court's cas-lwas No. 118. Glor v. Switzerland - 13444/04. Judgement 30.4.2009. http://hudoc.echr.coe.int/sites/eng/pages/search.aspx? $\mathrm{i}=001-92525$. Zugegriffen: 15.05 .2014 .

Fernandes, P. T., Salgado, P.C.B., Noronha, A.L.A., Barbosa, F. D., Souza, E. A.P., Sander, J. W., \& Li, L. M. (2007). Prejudice towards chronic diseases: comparison among epilepsy, AIDS and diabetes. Seizure, 16, 320-323.

Fraser, M.W., Richman, J.M., Galinski, M.J., \& Day, S.H. (2009). Intervention research. Developing social programs. New York: Oxford University Press.

Goffman, E. (1963). Stigma: notes on the management of spoiled identity. Englewood Cliffs: PrenticeHall.

Gredig, D. (2011). From research to practice: research-based intervention development in social work. Developing practice through cooperative knowledge production. European Journal of Social Work, 14(1), 53-70.

Griffith, R.G., \& Moses, R.G. (1993). Diabetes in the workplace. Employment experiences of young people with diabetes mellitus. The Medical Journal of Australia, 158(3), 169-171.

Grossman, Cynthia I./Stangl, Anne L. (2013). Global action to reduce HIV stigma and discrimination. Journal of the International AIDS Society, 16, (3Suppl 2), S. 18881. http://www.ncbi.nlm.nih.gov/ pmc/articles/PMC3833103/. https://doi.org/10.7448/IAS.16.3.18881. 
Huber, C. A., Schwenkglenks, M., Rapold, R., \& Reich, O. (2014). Epidemiology and costs of diabetes mellitus in Switzerland: an analysis of health care claims data, 2006 and 2011. BMC Endocrine Disorders. https://doi.org/10.1186/1472-6823-14-44.

Költringer, R. (1993). Gültigkeit von Umfragedaten. Wien: Böhlau.

Link, B. G., \& Phelan, J.C. (2001). Conceptualizing stigma. Annual Review of Sociology, 27, 363-385.

Nebiker-Pedrotti, P. M., Keller, U., Iselin, H.-U., Ruiz, J., Pärli, K., \& Caplazi, A. (2009). Perceived discrimination against diabetics in the work place and in work-related insurances in Switzerland. Swiss Medical Weekly, 139(7-8), 103-109.

Osborne, J.W., \& Costello, A. B. (2009). Best practices in exploratory factor analysis: four recommendations for getting the most from your analysis. Pan-Pacific Management Review, 12(2), 131-146.

Pärli, K. (2009). Rechtsgutachten „Diskriminierung von Diabetes-Patienten/innen “. Winterthur: ZHAW School of Management and Law.

Patton, M.Q. (2002). Qualitative research and evaluation methods (3. Aufl.). Thousand Oaks, London, New Delhi: SAGE.

Pescosolido, B. A., Martin, J. K., Lang, A., \& Olafsdottir, S. (2008). Rethinking theoretical approaches to stigma: a framework integrating normative influences on stigma (FINIS). Social Science \& Medicine, 67, 431-440.

Petrides, P., Petermann, F., Henrichs, H. R., Petzoldt, R., Rölver, K. M., Schidlmeier, A., Wendt, A., et al. (1995). Coping with employment discrimination against diabetics: trends in social medicine and social psychology. Patient Education Counseling, 26, 203-208.

Raemy, A., Gredig, D., \& Grillet, S. (2012). Diskriminiserungs- und Stigmatisierungserfahrungen von Personen mit Diabetes in der Schweiz. Eine qualitative Rekonstruktion von Stigmatisierung und Diskriminierung aus der Sicht der Betroffenen. Olten: Fachhochschule Nordwestschweiz FHNW.

Robinson, N., Yateman, N. A., Protopapa, L.E., \& Bush, L. (1990). Employment problems and diabetes. Diabetic Medicine, 7(1), 16-22.

Schabert, J., Browne, J. L., Mosely, K., \& Speight, J. (2013). Social stigma in diabetes. Patient, 6, 1-10.

Scherpenzeel, A.C., \& Saris, W.E. (1997). The validity and reliability of survey questions. A meta-analysis of MTMM studies. Sociological Methods and Research, 25, 341-383.

Schnell, R., Hill, P. B., \& Esser, E. (1999). Methoden der empirischen Sozialforschung. München: Oldenbourg.

Songer, T. J., Laporte, R. E., Dorman, J. S., Orchard, T. J., Becker, D. J., \& Drash, A. L. (1989). Employment spectrum of IDDM. Diabetes Care, 12, 615-622.

Strauss, A., \& Corbin, J. (1996). Grounded Theory: Grundlagen Qualitativer Sozialforschung. Weinheim: Beltz.

Tak-Ying Shiu, A., Kwan, Y.-M. J. J., \& Wong, Y.-M. R. (2003). Social stigma as barrier to diabetes selfmanagement: implications for multi-level interventions. Journal of Clinical Nursing, 12, 149-150.

Vishwanath, A. (2014). Negative public perceptions of juvenile diabetics: applying attribution theory to understand the public's stigmatizing views. Health Communication, 29(5), 516-526. https://doi.org/ 10.1080/10410236.2013.777685.

Voseckova, A., Truhlarova, Z., Levicka, J., Klimova, B., \& Kuca, K. (2017). Application of salutogenic concept in social work with diabetic patients. Social Work in Health Care, 56(4), 244-259. https:// doi.org/10.1080/00981389.2016.1265635.

Wardian, J., \& Sun, F. (2014). Factors associated with diabetes-related distress: implications for diabetes self-management. Social Work in Health Care, 53(4), 364-381. https://doi.org/10.1080/00981389. 2014.884038 .

Wens, J., Nicolucci, A., Kalra, S., Kulzer, B., Benedetti, M. M., Piana, N., et al. (2013). Societal discrimination and emotional well-being in people with diabetes: results from DAWN2. Diabetologia, 56, 458-S459.

Winkler, M. (2003). Theorie der Sozialpädagogik - eine Rekonstruktion. Zeitschrift für Sozialpädagogik, $1(1), 6-24$.

Witzel, A. (1985). Das problemzentrierte Interview. In G. Jüttemann (Hrsg.), Qualitative Forschung in der Psychologie. Grundfragen, Verfahrensweisen, Anwendungsfelder (S. 227-255). Weinheim, Basel: Beltz. 